Теорія Ймовір. та Матем. Статист. Вип. 77, 2007
Theor. Probability and Math. Statist.

No. 77, 2008, Pages 39-55

S 0094-9000(09)00746-7

Article electronically published on January 14, 2009

\title{
SUFFICIENT CONDITIONS FOR THE CONVERGENCE OF LOCAL-TIME TYPE FUNCTIONALS OF MARKOV APPROXIMATIONS
}

UDC 519.21

\author{
YU. M. KARTASHOV
}

\begin{abstract}
A sufficient condition is obtained for the weak convergence of additive functionals defined on a sequence of Markov chains $X_{n}$ approaching a Markov process $X$. The condition is expressed in terms of transient probabilities of the chains $X_{n}$. An application of the main result is given for the convergence on the Cantor set of local-time type functionals of random walks approaching an $\alpha$-stable process with index $\alpha \leq 1$.
\end{abstract}

\section{INTRODUCTION}

The aim of the paper is to find sufficient conditions for the convergence in distribution of functionals of stochastic processes approximating a homogeneous Markov process.

Suppose a sequence of stochastic processes $X_{n}, n \geq 1$, approximates in the Markov sense (see [1]) a homogeneous Markov process $X$. Let

$$
t_{k, n} \stackrel{\text { def }}{=} \frac{k}{n}
$$

We consider functionals $\phi_{n}$ of the following form:

$$
\phi_{n}^{s, t}\left(X_{n}\right) \stackrel{\text { def }}{=} \sum_{s \leq t_{k, n}<t} g_{n}\left(X_{n}\left(t_{k, n}\right)\right), \quad 0 \leq s<t,
$$

where $g_{n}$ are nonnegative Borel functions. The functionals $\phi_{n}$ are stepwise functions with respect to each of their time argument. Consider the polygonal lines constructed from these functionals:

$$
\begin{gathered}
\psi_{n}^{s, t}=\phi_{n}^{t_{j-1, n}, t_{k-1, n}}-(n s-j+1) \phi_{n}^{t_{j-1, n}, t_{j, n}}+(n t-k+1) \phi_{n}^{t_{k-1, n}, t_{k, n}}, \\
s \in\left[t_{j-1, n}, t_{j, n}\right), \quad t \in\left[t_{k-1, n}, t_{k, n}\right) .
\end{gathered}
$$

The lines $\psi_{n}$ are viewed as random elements in the space $C\left(\mathbb{T}, \mathbb{R}^{+}\right)$, where

$$
\mathbb{T} \stackrel{\text { def }}{=}\{(s, t) \mid 0 \leq s \leq t\} .
$$

We consider the problem of the weak convergence of $\psi_{n}$ in the space $C\left(\mathbb{T}, \mathbb{R}^{+}\right)$to the element

$$
\left\{\phi^{s, t},(s, t) \in \mathbb{T}\right\},
$$

2000 Mathematics Subject Classification. Primary 60J55, 60J45, 60F17.

Key words and phrases. Additive functional, characteristic of an additive functional, Markov approximation.

The work is supported by the Ministry of Science and Education of Ukraine, Project N GP/F13/0095. 
where $\phi$ is a Wiener $W$-functional of the limit process $X$. Theorem 1, the main result of the paper, contains sufficient conditions for such a convergence.

We show that this convergence follows from a condition that is a version of the local limit theorem for the processes $X_{n}$ and from an estimate for the distributions of sequential exit times from a set with a curvilinear boundary. This estimate is of its own importance.

The proof of the main result is based on Theorem 1 of the paper [2], where we considered a general setting for the weak convergence of functionals (1.1). The main assumption of Theorem 1 in 2 is the convergence of the characteristics of the functionals $\phi_{n}$ to the characteristic of the functional $\phi$. In this paper, we obtain sufficient conditions for the uniform convergence of the characteristics of $\phi_{n}$. The method of the proof in [2] is a modification of Dynkin's approach (see [3, Chapter 6]) to the convergence of $W$-functionals.

As an application of the main result, we consider the problem of the weak convergence of local-time type functionals of random walks on the Cantor set. The functionals coincide, up to a normalizing factor, with the measure of the time spent by a random polygonal line constructed from a random walk in a neighborhood of the Cantor set. The random walk is assumed to approximate an $\alpha$-stable process with $\alpha \leq 1$.

\section{Main RESUlt}

Let $(\mathfrak{X}, \rho)$ be a locally compact metric space. Consider processes $X$ and $X_{n}, n \geq 1$, defined on $\mathbb{R}^{+}$and whose phase space is $\mathfrak{X}$. We assume that $X$ is a homogeneous Markov process. Let the process $X_{n}$ be Markovian for every $n \geq 1$ if the time parameter set is reduced to the set $\left\{t_{k, n}, k \geq 0\right\} \cap[0, T]$, where $T$ is a fixed positive number.

The following definition from [1] is used in our main result.

Definition 1. A sequence $\left\{X_{n}\right\}$ approximates a process $X$ in the Markov sense if, for all $\gamma>0$ and $T>0$, there exist a number $K(\gamma, T) \in \mathbb{N}$ and a sequence of two-component processes $\left\{\hat{Y}_{n}=\left(\hat{X}_{n}, \hat{X}^{n}\right)\right\}$ defined on a different probability space and such that

(i) $\hat{X}_{n} \stackrel{d}{=} X_{n}, \hat{X}^{n} \stackrel{d}{=} X$;

(ii) the process $\hat{Y}_{n}$ as well as the processes $\hat{X}_{n}$ and $\hat{X}^{n}$ possess the Markov property at the points $t_{i K(\gamma, T), n}, i \in \mathbb{N}$, with respect to the flow $\left\{\hat{\mathcal{F}}_{t}^{n}=\sigma\left(\hat{Y}_{n}(s), s \leq t\right)\right\}$.

$$
\limsup _{n \rightarrow+\infty} \mathrm{P}\left(\sup _{i \leq \frac{T n}{K(\gamma, T)}} \rho\left(\hat{X}_{n}\left(t_{i K(\gamma, T), n}\right), \hat{X}^{n}\left(t_{i K(\gamma, T), n}\right)\right)>\gamma\right)<\gamma .
$$

Assume that the transient probabilities of the process $X_{n}$ have density for all $n$, that is,

$$
\mathrm{P}\left(X_{n}\left(t_{i+k, n}\right) \in d y \mid X_{n}\left(t_{i, n}\right)=x\right)=p_{n, k}(x, y) d y, \quad i \in \mathbb{Z}_{+}, k \in \mathbb{N}, x, y \in \mathfrak{X} .
$$

We also assume that the transient probability of the process $X$ has density:

$$
\mathrm{P}(X(t) \in d y \mid X(s)=x)=p_{t-s}(x, y) d y, \quad 0 \leq s<t \leq T, x, y \in \mathfrak{X} .
$$

Consider the functionals

$$
\phi_{n}^{s, t}\left(X_{n}\right) \stackrel{\text { def }}{=} \sum_{k: s \leq t_{k, n}<t} g_{n}\left(X_{n}\left(t_{k, n}\right)\right), \quad 0 \leq s<t, g_{n} \in \mathcal{B}\left(\mathfrak{X}, \mathbb{R}^{+}\right), n \geq 1 .
$$

We denote by $\mu_{n}(d u) \stackrel{\text { def }}{=} n g_{n}(u) d u$ the so-called symbols of the functionals $\phi_{n}$. Following Dynkin's approach ([3, Chapter 6]), consider

$$
f_{n}^{s, t}(x) \stackrel{\text { def }}{=} \mathrm{E}\left[\phi_{n}^{s, t}\left(X_{n}\right) \mid X_{n}(s)=x\right], \quad s=t_{i, n}, i \in \mathbb{Z}_{+}, 0 \leq s<t<T, x \in \mathfrak{X} .
$$

In what follows $f_{n}^{s, t}(x)$ is called the characteristic of $\phi_{n}$. 
We further assume that a $W$-functional (see [3]) $\phi=\phi(X)$ with characteristic $f$ is given. It is known (see [3, Chapter 6]) that

$$
\phi^{s, t}=\underset{\varepsilon \rightarrow 0+}{L_{2^{-}} \lim _{s}} \int_{s}^{t} \frac{1}{\varepsilon} f^{0, \varepsilon}(X(r)) d r
$$

This implies that

$$
f^{s, t}(x)=\lim _{\varepsilon \rightarrow 0+} \int_{s}^{t} \int_{\mathfrak{X}} p_{r}(x, y) \frac{1}{\varepsilon} f^{0, \varepsilon}(y) d y d r .
$$

We assume further that the measures $\varepsilon^{-1} f^{0, \varepsilon} d u$ converge weakly as $\varepsilon \rightarrow 0+$ to a finite measure $\mu$. We also assume that the characteristic $f$ admits the representation

$$
f^{s, t}(x)=\int_{0}^{t-s} \int_{\mathfrak{X}} p_{r}(x, y) \mu(d y) d r,
$$

where

$$
\int_{0}^{T}\left[\sup _{x \in \mathfrak{X}} \int_{\mathfrak{X}} p_{r}(x, y) \mu(d y)\right] d r<+\infty, \quad T>0 .
$$

Let $B(x, R) \equiv\{x \in \mathfrak{X} \mid \rho(x, y)<R\}$ and $\mathbb{I}_{n} \stackrel{\text { def }}{=}[0, T] \cap \frac{1}{n} \mathbb{Z}$.

Theorem 1. Assume that there are constants $\beta, \gamma, \theta, A_{0}>0$ and $\varepsilon \in(0,1)$ such that

(1) the trajectories of the processes $X_{n}$ are continuous and the sequence $\left\{X_{n}\right\}$ approximates the process $X$ in the Markov sense;

(2) there exists a constant $C_{\beta, \delta, \varepsilon}>0$ such that

$$
\sup _{x \in \mathbb{R}} \mathrm{P}_{x}\left(\rho\left(x, X_{n}(t)\right)>A t^{\delta}\right) \leq C_{\beta, \delta, \varepsilon} A^{-\beta} t^{\varepsilon}
$$

for all $n \geq 1, A>A_{0}$, and $t \in \mathbb{I}_{n}$

(3) $\sup _{x \in \mathfrak{X}} g_{n}(x) \rightarrow 0$ as $n \rightarrow \infty$;

(4) for a given $t_{0}>0$, the function $(t, x, y) \mapsto p_{t}(x, y)$ is uniformly continuous on $\left[t_{0},+\infty\right) \times \mathfrak{X}^{2}$ and such that

$$
\sup _{x \notin B(y, R)} p_{t}(x, y) \rightarrow 0, \quad R \rightarrow+\infty,
$$

for all $y \in \mathfrak{X}$ and $t>0$; moreover, let there exist a constant $C_{\gamma}>0$ such that

$$
\sup _{x, y \in \mathfrak{X}} p_{t}(x, y) \leq C_{\gamma} t^{-\gamma}, \quad t>0
$$

(5) there exists a sequence $\left\{\alpha_{k}\right\} \subset \mathbb{R}^{+}$converging to zero and such that

$$
\sup _{x, y \in \mathfrak{X}}\left|p_{n, k}(x, y)-p_{\frac{k}{n}}(x, y)\right| \leq \alpha_{k}\left(\frac{n}{k}\right)^{\gamma}, \quad n, k \in \mathbb{N} ;
$$

(6) the measures $\mu_{n}$ are finite, weakly converge to a measure $\mu$, and there are constants $C_{\theta}, c_{\theta}>0$ such that

$$
\mu_{n}(B(x, R)) \leq C_{\theta} R^{\theta}, \quad x \in \mathfrak{X}, n \in \mathbb{N}, R>c_{\theta} n^{-\delta} ;
$$

(7) the constants $\gamma, \delta$, and $\theta$ are such that

$$
\delta \theta+1>\gamma .
$$

Then the random polygonal lines $\psi_{n}$ constructed from the functionals $\phi_{n}$ converge in distribution to $\phi$ in the space $C\left(\mathbb{T}, \mathbb{R}^{+}\right)$.

Prior to the proof of the main result we consider an auxiliary result which is of its own interest. 


\section{Auxiliary Results on the exit times}

Assume that $Z$ is a homogeneous Markov process taking values in a locally compact metric space $(\mathfrak{X}, \rho)$ and defined on the set $\mathbb{I}_{n}$. As in the preceding section, let $t_{k, n} \stackrel{\text { def }}{=} k / n$.

For all $A, \delta>0$, and $n \in \mathbb{N}$, consider a sequence $\left\{\tau^{m, A, \delta}, m \geq 0\right\}$ of stopping times with respect to the filtration generated by the process $Z$ :

$$
\begin{gathered}
\tau^{0, A, \delta} \stackrel{\text { def }}{=} 0, \\
\tau^{m+1, A, \delta} \stackrel{\text { def }}{=} \inf \left\{t_{i, n}: t_{i, n}>\tau^{m, A, \delta}, \rho\left(Z\left(t_{i, n}\right), Z\left(\tau^{m, A, \delta}\right)\right)>A\left(t_{i, n}-\tau^{m, A, \delta}\right)^{\delta}\right\} .
\end{gathered}
$$

Lemma 1. Assume that the following form of condition (2) in Theorem 1 holds for the process $Z$ :

$$
\begin{aligned}
& \exists A_{0}, C, T, \beta, \delta>0, \varepsilon \in(0,1): \\
& \forall A \geq A_{0}, \forall t \in \mathbb{I}_{n} \quad \sup _{x \in \mathfrak{X}} \mathrm{P}\left(\rho(x, Z(t))>A t^{\delta} \mid Z(0)=x\right) \leq C A^{-\beta} t^{\varepsilon} .
\end{aligned}
$$

Then there exist constants $\widetilde{C}>0, \widetilde{C}_{1}>0$, and $A_{1}>0$ such that

$$
\forall A \geq A_{1}, \forall t \in \mathbb{I}_{n}, \forall m \geq 1 \quad \sup _{x \in \mathfrak{X}} \mathrm{P}\left(\tau^{m, A, \delta}<t \mid Z(0)=x\right) \leq \frac{\widetilde{C}\left(\widetilde{C}_{1} C A^{-\beta}\right)^{m}}{\Gamma^{\varepsilon}(m)} t^{m \varepsilon} .
$$

We split the proof of Lemma 1 in two steps. The first step is to obtain a bound for the distribution of the first exit time $\tau^{1, A, \delta}$. The second step is to estimate the distribution of the convolutions of $\tau^{1, A, \delta}$.

Lemma 2. If assumptions of Lemma 1 hold, then there exist constants $A_{2}>0$ and $\widetilde{C}>0$ such that

$$
\begin{aligned}
\forall A> & A_{2}, \forall t \in \mathbb{I}_{n} \\
& \sup _{x \in \mathfrak{X}} \mathrm{P}\left(\sup _{u: u \in(0, t) \cap \frac{1}{n} \mathbb{Z}}\left(\rho(x, Z(u))-A u^{\delta}\right)>0 \mid Z(0)=x\right) \leq \widetilde{C} t^{\varepsilon} A^{-\beta} .
\end{aligned}
$$

Proof. Put $k=t n$ and $N=\left[\log _{2} k\right]+1$. Then

$$
\begin{aligned}
& \mathrm{P}\left(\sup _{l=1, \ldots, k}\left(\rho\left(x, Z\left(t_{l, n}\right)\right)-A\left(t_{l, n}\right)^{\delta}\right)>0 \mid Z(0)=x\right) \\
& \quad \leq \mathrm{P}\left(\sup _{i=0, \ldots, N} \sup _{l=\left[\frac{n t}{2^{i+1}}\right], \ldots,\left[\frac{n t}{2^{i}}\right]}\left(\rho\left(x, Z\left(t_{l, n}\right)\right)-A\left(t_{l, n}\right)^{\delta}\right)>0 \mid Z(0)=x\right) \\
& \quad \leq \mathrm{P}\left(\sup _{i=0, \ldots, N} \sup _{l=\left[\frac{n t}{2^{2+1}}\right], \ldots,\left[\frac{n t}{2^{i}}\right]}\left(\rho\left(x, Z\left(t_{l, n}\right)\right)-A\left(t_{\left[\frac{n t}{2^{i+1}}\right], n}\right)^{\delta}\right)>0 \mid Z(0)=x\right) \\
& \quad \leq \sum_{i=0}^{N} P\left(\sup _{l=\left[\frac{n t}{\left.2^{2+1}\right], \ldots,\left[\frac{n t}{2^{i}}\right]}\right]} \rho\left(x, Z\left(t_{l, n}\right)\right)-A\left(t_{\left[\frac{n t}{2^{2+1}}\right], n}\right)^{\delta}>0 \mid Z(0)=x\right) \\
& \quad=\sum_{i=0}^{N} P\left(\sup _{l=\left[\frac{n t}{2^{i+1}}\right], \ldots,\left[\frac{n t}{2^{i}}\right]} \rho\left(x, Z\left(t_{l, n}\right)\right)-A\left(t_{\left[\frac{n t}{2^{2+1}}\right], n}\right)^{\delta}>0 \mid Z(0)=x\right) .
\end{aligned}
$$

Now we apply Skorokhod's inequality of Lemma 2 in [8, §9], where the proof is given for the case where the phase space is $\mathbb{R}$; the general case is treated similarly. Let $\xi_{j}$, 
$j=2, \ldots,\left[\frac{n t}{2^{i}}\right]-\left[\frac{n t}{2^{i+1}}\right]+1$, be equal to the values of the process at the points $\left[\frac{n t}{2^{i+1}}\right]$, $\left[\frac{n t}{2^{i+1}}\right]+1, \ldots,\left[\frac{n t}{2^{i}}\right]$, respectively, and let $\xi_{1} \stackrel{\text { def }}{=} 0$. Since

$$
\begin{aligned}
& \mathrm{P}(\rho\left.\left(Z\left(t_{l, n}\right), Z\left(t_{\left[\frac{n t}{2^{i}}\right], n}\right)\right) \geq \frac{A}{2}\left(t_{\left[\frac{n t}{2^{2+1}}\right], n}\right)^{\delta} \mid \mathcal{F}_{t_{l, n}}\right) \\
& \quad \mathrm{P}\left(\rho\left(Z\left(t_{l, n}\right), Z\left(t_{\left[\frac{n t}{2^{i}}\right], n}\right)\right) \geq \frac{A}{2}\left(t_{\left[\frac{n t}{2^{i+1}}\right], n}\right)^{\delta} \mid Z\left(t_{l, n}\right)\right) \\
& \quad \leq \mathrm{P}\left(\rho\left(Z\left(t_{l, n}\right), Z\left(t_{\left[\frac{n t}{2^{i}}\right], n}\right)\right) \geq \frac{A}{2}\left(t_{\left[\frac{n t}{2^{i+1}}\right], n}-t_{l, n}\right)^{\delta} \mid Z\left(t_{l, n}\right)\right) \\
& \quad \leq C 2^{\beta} A^{-\beta}\left(t_{\left[\frac{n t}{2^{2+1}}\right], n}-t_{l, n}\right)^{\varepsilon} \leq C 2^{\beta} A^{-\beta}\left(t_{\left[\frac{n t}{2^{i+1}}\right], n}\right)^{\varepsilon},
\end{aligned}
$$

we obtain the bound

$$
\begin{aligned}
& \sum_{i=0}^{N} \mathrm{P}\left(\sup _{l=\left[\frac{n t}{2^{i+1}}\right], \ldots,\left[\frac{n t}{2^{i}}\right]} \rho\left(x, Z\left(t_{l, n}\right)\right)>A\left(t_{\left[\frac{n t}{2^{i+1}}\right], n}\right)^{\delta} \mid Z(0)=x\right) \\
& \quad \leq C 2^{\beta} \sum_{i=0}^{N} A^{-\beta}\left(t_{\left[\frac{n t}{2^{i+1}}\right], n}\right)^{\varepsilon} \leq \widetilde{C} t^{\varepsilon} A^{-\beta}
\end{aligned}
$$

for all $t \in \mathbb{I}_{n}$ and all sufficiently large $A$. This completes the proof of Lemma 2 .

Lemma 3. Let a random variable $\tau$ assume values in $\frac{1}{n} \mathbb{Z}^{+}$. We denote by $\tau_{m}$ a random variable whose distribution is the $m$-th convolution of the distribution of $\tau$. Let, for some $T>0$ and $\varepsilon \in(0,1)$,

$$
\mathrm{P}\left(\tau \leq t_{k, n}\right) \leq C_{1} t_{k, n}^{\varepsilon} \quad \text { for all } k=1, \ldots,[n T] .
$$

Then there are $C_{2}=C_{2}(\varepsilon)>0$ and $C_{3}=C_{3}(\varepsilon)$ such that

$$
\mathrm{P}\left(\tau_{m} \leq t_{k, n}\right) \leq \frac{C_{2}\left(C_{3} C_{1}\right)^{m}}{\Gamma^{\varepsilon}(m)} t_{k, n}^{m \varepsilon} \quad \text { for all } k=1, \ldots,[n T] .
$$

Proof. Assume that

$$
\exists C_{m}>0: \quad \mathrm{P}\left(\tau_{m} \leq t_{k, n}\right) \leq C_{m} t_{k, n}^{m \varepsilon}, \quad k=1, \ldots,[n T],
$$

for some $m \geq 1$. To estimate the distribution function of the random variable $\tau_{m+1}$ we apply a discrete analogue of the integration by parts formula:

$$
\begin{aligned}
\mathrm{P}\left(\tau_{m+1} \leq t_{k, n}\right) & =\sum_{i=0}^{k} \mathrm{P}\left(\tau_{m} \leq t_{k, n}-t_{i, n}\right) \mathrm{P}\left(\tau_{1}=t_{i, n}\right) \\
& =\sum_{i=0}^{k} C_{m}\left(t_{k, n}-t_{i, n}\right)^{m \varepsilon}\left(\mathrm{P}\left(\tau_{1} \leq t_{i, n}\right)-\mathrm{P}\left(\tau_{1} \leq t_{i-1, n}\right)\right) \\
& =\sum_{i=0}^{k} C_{m}\left(t_{k-i, n}\right)^{m \varepsilon} \mathrm{P}\left(\tau_{1} \leq t_{i, n}\right)-\sum_{i=-1}^{k-1} C_{m}\left(t_{k-i-1, n}\right)^{m \varepsilon} \mathrm{P}\left(\tau_{1} \leq t_{i, n}\right) \\
& =\sum_{i=0}^{k-1} C_{m} C_{1}\left[\left(t_{k-i, n}\right)^{m \varepsilon}-\left(t_{k, n}-t_{i+1, n}\right)^{m \varepsilon}\right]\left(t_{i, n}\right)^{\varepsilon} \\
& =\left(t_{k, n}\right)^{(m+1) \varepsilon} C_{m} C_{1} \sum_{i=0}^{k-1}\left[\left(1-\frac{i}{k}\right)^{m \varepsilon}-\left(1-\frac{i+1}{k}\right)^{m \varepsilon}\right]\left(\frac{i}{k}\right)^{\varepsilon}
\end{aligned}
$$


In what follows we consider two cases, namely $m \varepsilon \geq 1$ and $m \varepsilon<1$. First let $m \varepsilon<1$. Then

$$
\begin{aligned}
\mathrm{P}\left(\tau_{m+1} \leq t_{k, n}\right) & =\left(t_{k, n}\right)^{(m+1) \varepsilon} C_{m} C_{1} \sum_{i=0}^{k-1}\left[\left(1-\frac{i}{k}\right)^{m \varepsilon}-\left(1-\frac{i+1}{k}\right)^{m \varepsilon}\right]\left(\frac{i}{k}\right)^{\varepsilon} \\
& \leq\left(t_{k, n}\right)^{(m+1) \varepsilon} C_{m} C_{1} \sum_{i=0}^{k-1}\left[\left(1-\frac{i}{k}\right)^{m \varepsilon}-\left(1-\frac{i+1}{k}\right)^{m \varepsilon}\right] \\
& =\left(t_{k, n}\right)^{(m+1) \varepsilon} C_{m} C_{1} .
\end{aligned}
$$

Therefore

$$
\mathrm{P}\left(\tau_{m} \leq t_{k, n}\right) \leq C_{1}^{m}\left(t_{k, n}\right)^{m \varepsilon} \quad \text { for all } m \in \mathbb{Z} \cap\left[1, \frac{1}{\varepsilon}\right], k=1, \ldots,[n T] .
$$

Now we consider the case of $m \varepsilon \geq 1$. Then

$$
\begin{aligned}
\left(t_{k, n}\right)^{(m+1) \varepsilon} C_{1} C_{m} \sum_{i=0}^{k-1}\left[\left(1-\frac{i}{k}\right)^{m \varepsilon}-\left(1-\frac{i+1}{k}\right)^{m \varepsilon}\right]\left(\frac{i}{k}\right)^{\varepsilon} \\
\quad \leq C_{1} C_{m}\left(t_{k, n}\right)^{(m+1) \varepsilon} \int_{0}^{1} u^{\varepsilon} d\left[-(1-u)^{m \varepsilon}\right]=C_{1} C_{m}\left(t_{k, n}\right)^{(m+1) \varepsilon} m \varepsilon B(m \varepsilon, \varepsilon+1) \\
\quad=C_{1} C_{m}\left(t_{k, n}\right)^{(m+1) \varepsilon}\left(m \varepsilon \frac{\Gamma(m \varepsilon) \Gamma(\varepsilon+1)}{\Gamma(m \varepsilon+\varepsilon+1)}\right) \\
\leq C_{1} C_{m}\left(t_{k, n}\right)^{(m+1) \varepsilon} \frac{\Gamma(m \varepsilon+1)}{\Gamma(m \varepsilon+1+\varepsilon)} \leq C_{1} C_{m}\left(t_{k, n}\right)^{(m+1) \varepsilon} \frac{1}{m^{\varepsilon}}
\end{aligned}
$$

We have used the following bound:

$$
\begin{aligned}
\frac{\Gamma(m \varepsilon+1)}{\Gamma(m \varepsilon+1+\varepsilon)} & =\frac{\Gamma(m \varepsilon-[m \varepsilon]+1)}{\Gamma(m \varepsilon+\varepsilon-[m \varepsilon]+1)} \cdot \prod_{i=0}^{[m \varepsilon]-1}\left(\frac{m \varepsilon-i}{m \varepsilon+\varepsilon-i}\right) \\
& \leq \exp \left[\sum_{i=0}^{[m \varepsilon]}\left(\frac{m \varepsilon-i}{m \varepsilon+\varepsilon-i}-1\right)\right] \leq \exp \left[-\varepsilon \sum_{i=1}^{[m \varepsilon]-1} \frac{1}{i}\right] \\
& \leq \exp [-\varepsilon \ln (m \varepsilon)]=\frac{\varepsilon^{-\varepsilon}}{m^{\varepsilon}} .
\end{aligned}
$$

Combining this bound with the bound obtained in the first case, we conclude that there are constants $C_{2}=\left(\Gamma\left(\varepsilon^{-1}\right)\right)^{\varepsilon}>0$ and $C_{3}=\left(\varepsilon^{-1}\right)^{\varepsilon}>0$ such that

$$
\mathrm{P}\left(\tau_{m} \leq t_{k, n}\right) \leq \frac{C_{2}\left(C_{3} C_{1}\right)^{m}}{\Gamma^{\varepsilon}(m)}\left(t_{k, n}\right)^{m \varepsilon} \quad \forall m \geq 1, \forall k=1, \ldots,[n T] .
$$

This result completes the proof of Lemma 3 .

Lemma 2 together with Lemma 3 proves Lemma 1.

\section{The proof of Theorem 1}

The following assertion is used to prove the convergence of functionals.

Proposition 1 ([2, Theorem 1]). Assume that a sequence $X_{n}$ approximates in the Markov sense a homogeneous Markov process $X$. Let the functionals $\left\{\phi_{n} \equiv \phi_{n}\left(X_{n}\right)\right\}$ be of the form (1.1). 
Assume that

(1) the functions $g_{n}(\cdot)$ are bounded on $\mathfrak{X}$ and converge uniformly to zero, that is,

$$
\delta_{n} \stackrel{\text { def }}{=} \sup _{x \in \mathfrak{X}} g_{n}(x) \rightarrow 0, \quad n \rightarrow \infty ;
$$

(2) there exists a function $f$ that is the characteristic (in the sense of [3, Chapter 6]) of a $W$-functional $\phi=\phi(X)$ of the limit Markov process $X$ and such that

$$
\sup _{s=\frac{i}{n}, t \in(s, T)}\left\|f_{n}^{s, t}(\cdot)-f^{s, t}(\cdot)\right\| \rightarrow 0, \quad n \rightarrow \infty,
$$

for all $T>0$, where $\|f(\cdot)\| \equiv \sup _{x \in \mathfrak{X}}|f(x)|$;

(3) the limit function $f$ is uniformly continuous with respect to the argument $x$, that is, for all $T>0$,

$$
\sup _{0 \leq s \leq t<T}\left|f^{s, t}\left(x^{\prime}\right)-f^{s, t}\left(x^{\prime \prime}\right)\right| \rightarrow 0, \quad\left|x^{\prime}-x^{\prime \prime}\right| \rightarrow 0 .
$$

Then the convergence in distribution holds in the space $C\left(\mathbb{T}, \mathbb{R}^{+}\right)$for the random polygonal lines $\psi_{n}$ constructed from the functionals $\phi_{n}$, that is,

$$
\psi_{n}\left(X_{n}\right) \Rightarrow \phi(X) \equiv\left\{\phi^{s, t}(X),(s, t) \in \mathbb{T}\right\} .
$$

Note that the assumptions of Theorem 1 imply condition (1) of Proposition 2 below.

Condition (3) follows from inequality (2.2) and assumption (4) of Theorem 1, Indeed, let $\varepsilon>0$ be fixed. Consider $r_{0}$ such that

$$
\int_{0}^{t_{0}}\left[\sup _{x \in \mathfrak{X}} \int_{\mathfrak{X}} p_{r}(x, y) \mu(d y)\right] d r<\frac{\varepsilon}{4} .
$$

Choose $\delta>0$ such that

$$
\mu(\mathfrak{X}) T \sup _{\substack{x_{1}, x_{2}, y \in \mathfrak{X}, r \geq r_{0}, \rho\left(x_{1}, x_{2}\right)<\delta}}\left|p_{r}\left(x_{1}, y\right)-p_{r}\left(x_{2}, y\right)\right|<\frac{\varepsilon}{2} .
$$

Taking into account the inequality

$$
\begin{aligned}
\left|f^{s, t}\left(x_{1}\right)-f^{s, t}\left(x_{2}\right)\right| \leq & \int_{0}^{r_{0}} \int_{\mathfrak{X}}\left(p_{r}\left(x_{1}, y\right)+p_{r}\left(x_{2}, y\right)\right) \mu(d y) d r \\
& +\int_{r_{0}}^{T} \int_{\mathfrak{X}}\left|p_{r}\left(x_{1}, y\right)-p_{r}\left(x_{2}, y\right)\right| \mu(d y) d r
\end{aligned}
$$

we get

$$
\sup _{s, t \in[0, T]} \sup _{\substack{x_{1}, x_{2} \in \mathfrak{X}, \rho\left(x_{1}, x_{2}\right)<\delta}}\left|f^{s, t}\left(x_{1}\right)-f^{s, t}\left(x_{2}\right)\right| \leq \varepsilon .
$$

It remains to check condition (2). We show that

$$
f_{n}^{s, t}(x) \rightrightarrows f^{s, t}(x), \quad n \rightarrow \infty .
$$

To prove (4.1) we proceed as follows. Consider the functionals $\hat{\phi}_{n, A}^{s, t}$ defined by

$$
\hat{\phi}_{n, A}^{s, t}=\sum_{k=[s n]-1}^{[t n]} \phi_{n}^{s \vee t_{k, n}, t \wedge t_{k+1, n}} \chi_{\left\{\rho\left(X_{n}\left(t_{k, n}\right), X_{n}(s)\right)<A\left|t_{k, n}-s\right|^{\delta}\right\}} .
$$


YU. M. KARTASHOV

The expectations of $\hat{\phi}_{n, A}^{s, t}$ are estimated from above with the help of conditions (5) and (6) of Theorem 1, namely, we have

$$
\begin{aligned}
\mathrm{E}\left[\hat{\phi}_{n, A}^{s, t} \mid X_{n}(s)=x\right] & =\mathrm{E}_{x} \sum_{i=s n-1}^{t n} \phi_{n}^{t_{i, n}, t_{i+1, n}} \chi_{\left\{\rho\left(X_{n}\left(t_{i, n}\right), X_{n}(s)\right)<A\left|t_{i, n}-s\right|^{\delta}\right\}} \\
& \leq g_{n}(x)+\frac{1}{n} \sum_{i=1}^{n t-n s-1} \int_{B\left(x, A\left(\frac{i}{n}\right)^{\delta}\right)} p_{n, i}(x, y) \mu_{n}(d y) \\
& \leq \delta_{n}+C_{\gamma}+\frac{\alpha}{n} \sum_{i=1}^{n t-n s-1}\left(\frac{i}{n}\right)^{-\gamma} \mu_{n}\left(B\left(x, A\left(\frac{i}{n}\right)^{\delta}\right)\right) \\
& \leq \delta_{n}+C_{\gamma}+\frac{\alpha}{n} \sum_{i=1}^{n t-n s-1} C_{\theta} A^{\theta}\left(\frac{i}{n}\right)^{-\gamma+\delta \theta}<B_{1}+B_{2}(T) A^{\theta}
\end{aligned}
$$

for all $s, t \in \mathbb{I}_{n}$ such that $s<t$ and for some positive constants $B_{1}, B_{2}(T), A>c_{\theta}$, and all $x \in \mathfrak{X}$, where $\alpha \stackrel{\text { def }}{=} \sup _{k} \alpha_{k}$.

Using the inequality

$$
\begin{aligned}
\sup _{x \in \mathfrak{X}} \mathrm{E} & {\left[\phi_{n}^{s, t} \chi_{\left\{\forall i: n s \leq i \leq n t, \rho\left(X_{n}\left(t_{i, n}\right), X_{n}(s) \mid\right)<A\left|t_{i, n}-s\right|^{\delta}\right\}} \mid X_{n}(s)=x\right] } \\
\leq & \sup _{x \in \mathfrak{X}} \mathrm{E}\left[\hat{\phi}_{n, A}^{s, t} \mid X_{n}(s)=x\right]<\infty
\end{aligned}
$$

we get a bound for the expectation of the functionals $\phi_{n, A}^{s, t}$ on the set of $\omega$ for which the trajectory of the process $X_{n}$ belongs to the domain

$$
\left\{(t, u): t \geq 0,|u| \leq t^{\delta}\right\} .
$$

We apply Lemma 1 to estimate the expectation of the functionals in the general case.

Let

$$
\begin{gathered}
\tau_{n}^{0, A, \delta} \stackrel{\text { def }}{=} s, \quad \tau^{0, A, \delta} \stackrel{\text { def }}{=} s, \\
\tau_{n}^{m+1, A, \delta} \stackrel{\text { def }}{=} \inf \left\{t_{u, n} \in[0, T]: t_{u, n}>\tau_{n}^{m, A, \delta},\right. \\
\left.\quad \rho\left(X_{n}\left(t_{u, n}\right), X_{n}\left(\tau_{n}^{m, A, \delta}\right)\right)>A\left(t_{u, n}-\tau_{n}^{m, A, \delta}\right)^{\delta}\right\}, \\
\tau^{m+1, A, \delta} \stackrel{\text { def }}{=} \inf \left\{z \in[0, T]: z>\tau^{m, A, \delta}, \rho\left(X(z), X\left(\tau^{m, A, \delta}\right)\right)>A\left(z-\tau^{m, A, \delta}\right)^{\delta}\right\} .
\end{gathered}
$$

Since the constants $A, \widetilde{C}$, and $\widetilde{C}_{1}$ in Lemma 1 depend on $\beta, \delta$, and $\varepsilon$ and do not depend on the process $Z$, we obtain

$$
\begin{aligned}
\exists A_{1}, C_{1}, C_{2}>0: \quad \forall m, n \geq 1, \forall s, t \in \mathbb{I}_{n}, \forall A>A_{1} \\
\sup _{x \in \mathcal{X}} \mathrm{P}_{x}\left(\tau_{n}^{m, A, \delta}<t\right) \leq \frac{C_{1}\left(C_{2} A^{-\beta}\right)^{m}}{\Gamma^{\varepsilon}(m)}(t-s)^{m \varepsilon} .
\end{aligned}
$$

Now we prove the inequality

$$
\begin{aligned}
\forall m \geq 1, \forall s, t \in(0, T), \forall A>A_{1} \\
\sup _{x \in \mathfrak{X}} \mathrm{P}_{x}\left(\tau^{m, A, \delta}<t\right) \leq \frac{C_{1}\left(C_{2} A^{-\beta}\right)^{m}}{\Gamma^{\varepsilon}(m)}(t-s)^{m \varepsilon} .
\end{aligned}
$$

First we check that

$$
\begin{aligned}
\forall n \geq 1, \forall A>A_{0}, \forall t \in(0, T) \cap \mathbb{Q} \\
\sup _{x \in \mathbb{R}} \mathrm{P}_{x}\left(\rho(x, X(t))>A t^{\delta}\right) \leq C_{\beta, \delta, \varepsilon} A^{-\beta} t^{\varepsilon} .
\end{aligned}
$$


Fix $\gamma>0$ and recall that $X_{n}$ approximates in the Markov sense the process $X$. Consider a sequence $\left(\widehat{X}^{n}, \widehat{X}_{n}\right)$ satisfying the conditions listed in Definition 1 . For all $t \in(0, T)$, $x \in \mathfrak{X}$, and $n \geq 1$, we have

$$
\begin{aligned}
\mathrm{P}_{x}\left(\rho(x, X(t))>A t^{\delta}+\gamma\right) & =\mathrm{P}_{x}\left(\rho\left(x, \widehat{X}^{n}(t)\right)>A t^{\delta}+\gamma\right) \\
& \leq \mathrm{P}_{x}\left(\rho\left(x, \widehat{X}_{n}(t)\right)>A t^{\delta}\right)+\mathrm{P}_{x}\left(\rho\left(\widehat{X}^{n}(t), \widehat{X}_{n}(t)\right)>\gamma\right) \\
& <C_{\beta, \delta, \varepsilon} A^{-\beta} t^{\varepsilon}+\mathrm{P}_{x}\left(\rho\left(\widehat{X}^{n}(t), \widehat{X}_{n}(t)\right)>\gamma\right) .
\end{aligned}
$$

Let $t=k_{t} / n_{t}$ for $k_{t}, n_{t} \in \mathbb{N}$, let $N_{n} \stackrel{\text { def }}{=} n \cdot n_{t} \cdot k(\gamma, T)$, and let $K_{n} \stackrel{\text { def }}{=} n \cdot k_{t}, n \geq 1$. Then

$$
\begin{aligned}
\mathrm{P}_{x} & \left(\rho\left(\widehat{X}^{N_{n}}\left(\frac{k_{t}}{n_{t}}\right), \widehat{X}_{N_{n}}\left(\frac{k_{t}}{n_{t}}\right)>\gamma\right)\right) \\
& =\mathrm{P}_{x}\left(\rho\left(\widehat{X}^{N_{n}}\left(k(\gamma, T) \frac{K_{n}}{N_{n}}\right), \widehat{X}_{N_{n}}\left(k(\gamma, T) \frac{K_{n}}{N_{n}}\right)>\gamma\right)\right) \\
& \leq \mathrm{P}_{x}\left(\sup _{i \in \mathbb{Z}^{+}: \frac{i k(\gamma, T)}{N_{n}} \in[0, T]} \rho\left(\widehat{X}^{N_{n}}\left(k(\gamma, T) \frac{i}{N_{n}}\right), \widehat{X}_{N_{n}}\left(k(\gamma, T) \frac{i}{N_{n}}\right)>\gamma\right)\right) \leq \gamma
\end{aligned}
$$

for sufficiently large $n$. Thus

$$
\mathrm{P}_{x}\left(\rho(x, X(t))>A t^{\delta}+\gamma\right)<\gamma+C_{\beta, \delta, \varepsilon} A^{-\beta} t^{\varepsilon} .
$$

Letting $\gamma \rightarrow 0$ we prove (4.3).

Consider the random variables

$$
\begin{gathered}
\widehat{\tau}_{n}^{0, A, \delta} \stackrel{\text { def }}{=} s, \\
\widehat{\tau}_{n}^{m+1, A, \delta} \stackrel{\text { def }}{=} \inf \left\{t_{u, n} \in[0, T]: t_{u, n}>\widehat{\tau}_{n}^{m, A, \delta},\right. \\
\left.\rho\left(X\left(t_{u, n}\right), X\left(\widehat{\tau}_{n}^{m, A, \delta}\right)\right)>A\left(t_{u, n}-\widehat{\tau}_{n}^{m, A, \delta}\right)^{\delta}\right\} .
\end{gathered}
$$

Since

$$
\mathrm{P}\left(\tau^{m, A, \delta}<t\right)=\lim _{n \rightarrow \infty} \mathrm{P}\left(\widehat{\tau}_{n}^{m, A, \delta}<t\right),
$$

Lemma 1 proves (4.2).

For $s, t \in \frac{1}{n} \mathbb{N}$ such that $s<t$, we represent $\phi_{n}^{s, t}$ as follows:

$$
\phi_{n}^{s, t}=\sum_{m=0}^{\infty} \phi_{n}^{\tau_{m}^{n, \delta}, t \wedge \tau_{m+1}^{n, \delta}} \chi_{\left\{\tau_{n}^{m, A, \delta}<t\right\}} .
$$

Then

$$
\begin{aligned}
& \mathrm{E}_{x} \phi_{n}^{\tau_{n}^{m, A, \delta}, \tau_{n}^{m+1, A, \delta} \wedge t} \chi_{\left\{\tau_{n}^{m, A, \delta}<t\right\}}=\mathrm{E}_{x}\left(\chi_{\left\{\tau_{n}^{m, A, \delta}<t\right\}} \mathrm{E}_{x}\left[\phi_{n}^{\tau_{n}^{m, A, \delta}, \tau_{n}^{m+1, A, \delta} \wedge t} \mid \mathcal{F}_{\tau_{n}^{m, A, \delta}}\right]\right) \\
& \quad=\mathrm{E}_{x}\left(\chi_{\left\{\tau_{n}^{m, A, \delta}<t\right\}} \mathrm{E}_{X_{n}\left(\tau_{n}^{m, A, \delta}\right)}\left[\hat{\phi}_{n}^{s, \tau_{n}^{m+1, A, \delta} \wedge t-\tau_{n}^{m, A \delta}+s}\right]\right) \\
& \leq \mathrm{P}_{x}\left(\tau_{n}^{m, A, \delta}<t\right) \sup _{x \in \mathfrak{X}} \mathrm{E}_{x} \hat{\phi}_{n}^{s, t} .
\end{aligned}
$$

Now we apply Lemma 1 to the random variables $\tau_{n}^{m, 1, \delta}-s$ for fixed $n, \delta$, and

$$
A=A_{2}>\max \left(1, A_{0}, A_{1}\right) .
$$


This allows us to obtain an upper bound for the expectation of the increment of the functional in the whole interval $(s, t)$ :

$$
\begin{aligned}
\mathrm{E} \phi_{n}^{s, t} & \leq \sup _{x \in \mathfrak{X}} \mathrm{E}_{x} \hat{\phi}_{n}^{s, t} \sum_{m=0}^{\infty} \mathrm{P}\left(\tau_{n}^{m, A_{2}, \delta}<t\right) \\
& \leq\left(B_{1}+B_{2}(T) A_{2}^{\theta}\right) \sum_{m=0}^{\infty} \frac{C_{1}\left(C_{2} A_{2}^{-\beta}\right)^{m}}{\Gamma(m)^{\varepsilon}}(t-s)^{m \varepsilon} \leq B_{3}(T) .
\end{aligned}
$$

In what follows we need a bound for the conditional second moment of the functional $\phi_{n}^{s, t}$, namely for

$$
\mathrm{E}\left[\left(\phi_{n}^{s, t}\right)^{2} \mid X_{n}(s)=x\right]
$$

We expand the second moment similarly to the case of the first moment and apply the strong Markov property:

$$
\begin{aligned}
\mathrm{E}_{x}\left(\phi_{n}^{s, t}\right)^{2}= & \mathrm{E}_{x}\left(\sum_{i=0}^{\infty} \phi_{n}^{\tau_{n}^{m, A_{2}, \delta}, t \wedge \tau_{m+1}^{n, \delta}} \chi_{\left\{\tau_{n}^{m, A_{2}, \delta}<t\right\}}\right)^{2} \\
= & 2 \mathrm{E}_{x} \sum_{i<j}^{\infty} \phi^{\tau_{n}^{i, A_{2}, \delta}, \tau_{n}^{i+1, A_{2}, \delta} \wedge t} \phi^{\tau_{n}^{j, A_{2}, \delta}, \tau_{n}^{j+1, A_{2}, \delta} \wedge t} \chi_{\left\{\tau_{n}^{j, A_{2}, \delta}<t\right\}} \\
& +\sum_{i=0}^{\infty} \mathrm{E}_{x}\left(\phi^{\tau_{n}^{i, A_{2}, \delta}, \tau_{n}^{i+1, A_{2}, \delta} \wedge t} \chi_{\left\{\tau_{n}^{i, A_{2}, \delta}<t\right\}}\right)^{2} \\
\leq & 2\left(\sup _{x \in \mathfrak{X}} \mathrm{E}_{x} \hat{\phi}_{n}^{s, t}\right)^{2} \sum_{i=0}^{\infty} \mathrm{P}_{x}\left(\tau_{n}^{i, A_{2}, \delta}<t\right)+\sum_{i=0}^{\infty} \mathrm{E}_{x}\left(\phi^{\tau_{n}^{i, A_{2}, \delta}, \tau_{n}^{i, 1 \delta} \wedge t} \chi_{\left\{\tau_{n}^{i, A_{2}, \delta}<t\right\}}\right)^{2} \\
\leq & 2\left(\sup _{x \in \mathfrak{X}} \mathrm{E}_{x} \hat{\phi}_{n}^{s, t}\right)^{2} \sum_{i=0}^{\infty} \mathrm{P}_{x}\left(\tau_{n}^{i, A_{2}, \delta}<t\right)+\sup _{x \in \mathcal{X}} \mathrm{E}_{x}\left[\left(\hat{\phi}_{n}^{s, t}\right)^{2}\right] \sum_{i=0}^{\infty} \mathrm{P}_{x}\left(\tau_{n}^{i, A_{2}, \delta}<t\right) \\
\leq & 4\left(\sup _{x \in \mathfrak{X}} \mathrm{E}_{x} \hat{\phi}_{n}^{s, t}\right)^{2} \sum_{i=0}^{\infty} \mathrm{P}_{x}\left(\tau_{n}^{i, A_{2}, \delta}<t\right) \\
& +\sup _{x \in \mathfrak{X}} \mathrm{E}_{x} \sum_{i=n s}^{n t}\left[\left(\hat{\phi}_{n}^{i / n,(i+1) / n}\right)^{2}\right] \sum_{i=0}^{\infty} \mathrm{P}_{x}\left(\tau_{n}^{i, A_{2}, \delta}<t\right) \\
\leq & 4\left(\sup _{x \in \mathfrak{X}} \mathrm{E}_{x} \hat{\phi}_{n}^{s, t}\right)^{2} \sum_{i=0}^{\infty} \mathrm{P}_{x}\left(\tau_{n}^{i, A_{2}, \delta}<t\right)+\delta_{n}\left(\sup _{x \in \mathfrak{X}} \mathrm{E}_{x} \hat{\phi}_{n}^{s, t}\right) \sum_{i=0}^{\infty} \mathrm{P}_{x}\left(\tau_{n}^{i, A_{2}, \delta}<t\right) \\
\leq & 4\left(B_{1}+B_{2}(T) A_{2}^{\theta}\right) B_{3}(T)+\delta_{n} B_{3}(T) \leq B_{4}(T) .
\end{aligned}
$$

A corresponding bound for the second moment of the functionals $\phi^{s, t}$ can be obtained similarly:

$$
\sup _{x \in \mathfrak{X}} \mathrm{E}_{x}\left(\phi^{s, t}\right)^{2} \leq B_{4}(T) .
$$

Now we prove the uniform convergence of the characteristics. Denote by $D_{n, A}^{s, t}$ the event $\left\{\tau_{n}^{1, A, \delta} \geq t\right\}$. Consider a nonincreasing Lipschitz function $\Psi: \mathbb{R}^{+} \rightarrow[0,1]$ such that $\Psi([0,1])=\{1\}$ and $\Psi([2,+\infty))=\{0\}$ and put

$$
\Psi_{r}(x, y)=\Psi\left(r^{-1} \cdot|x-y|\right), \quad r>0, x, y \in \mathfrak{X}, \quad \Psi_{0} \equiv 1 .
$$

Given an arbitrary $r_{0}>0$, the function $\Psi:(r, x, y) \mapsto \Psi_{r}(x, y)$ is uniformly continuous on $\left[r_{0},+\infty\right) \times \mathfrak{X}^{2}$. 
Let $s \leq t \leq T$ and $A>A_{0}$ be fixed. We represent $\phi_{n}^{s, t}$ in the form $\phi_{n}^{s, t}=\eta_{n, A}^{s, t}+\zeta_{n, A}^{s, t}$, where

$$
\eta_{n, A}^{s, t}=\frac{1}{n} \sum_{s \leq \frac{k}{n}<t} n g_{n}\left(X_{n}\left(t_{k, n}\right)\right) \Psi_{A\left(t_{k, n}-s\right)^{\delta}}\left(X_{n}(s), X_{n}\left(t_{k, n}\right)\right) .
$$

If $k$ is such that $s \leq t_{k, n}<t$, then

$$
\rho\left(X_{n}(s), X_{n}\left(t_{k, n}\right)\right) \leq A\left(t_{k, n}-s\right)^{\delta} \Rightarrow \Psi_{A\left(t_{k, n}-s\right)^{\delta}}\left(X_{n}(s), X_{n}\left(t_{k, n}\right)\right)=1
$$

on the set $D_{n, A}^{s, t}$, whence $\left\{\phi_{n}^{s, t}=\eta_{n, A}^{s, t}\right\} \supset D_{n, A}^{s, t}$ and

$$
\left\{\zeta_{n, A}^{s, t} \neq 0\right\} \subset \Omega \backslash D_{n, A}^{s, t} .
$$

Using the bound for the second moment of the functionals $\phi_{n}$ and the inequality

$$
0 \leq \zeta_{n, A}^{s, t} \leq \phi_{n}^{s, t}
$$

we prove the estimate

$$
\begin{aligned}
\mathrm{E}\left[\zeta_{n, A}^{s, t} \mid X_{n}(s)=x\right] & \leq \mathrm{E}\left[\left(\phi_{n}^{s, t}\right)^{2} \mid X_{n}(s)=x\right]^{1 / 2}\left[\mathrm{P}\left(\Omega \backslash D_{n, A}^{s, t} \mid X_{n}(s)=x\right)\right]^{1 / 2} \\
& \leq B_{5}(T) A^{-\beta / 2}
\end{aligned}
$$

Similarly $\phi^{s, t}=\eta_{A}^{s, t}+\zeta_{A}^{s, t}$, where

$$
\begin{gathered}
\eta_{A}^{s, t}=\int_{s}^{t} \Psi_{A(r-s)^{\delta}}(X(s), X(r)) d \phi^{s, r}, \\
\mathrm{E}\left[\zeta_{A}^{s, t} \mid X(s)=x\right] \leq B_{6}(T) A^{-\beta / 2} .
\end{gathered}
$$

Hence

$$
\begin{gathered}
\left|\mathrm{E}\left[\eta_{n, A}^{s, t} \mid X_{n}(s)=x\right]-\mathrm{E}\left[\eta_{A}^{s, t} \mid X(s)=x\right]\right| \\
\leq g_{n}(x)+\mid \frac{1}{n} \sum_{k=1}^{] n(t-s)[-1} \int_{\mathfrak{X}} p_{k, n}(x, y) \Psi_{A\left(\frac{k}{n}\right)^{\delta}}(x, y) \mu_{n}(d y) \\
\quad-\int_{0}^{t-s} \int_{\mathfrak{X}} p_{r}(x, y) \Psi_{A r^{\delta}}(x, y) \mu(d y) d r \mid \\
\leq \delta_{n}+\Delta_{n}^{1}(x, A, s, t)+\Delta_{n}^{2}(x, A, s, t)+\Delta_{n}^{3}(x, A, s, t),
\end{gathered}
$$

where $] z[\equiv \min \{N \in \mathbb{Z}, N \geq z\}$,

$$
\begin{gathered}
\Delta_{n}^{1}(x, A, s, t)=\left|\frac{1}{n} \sum_{k=1}^{] n(t-s)[-1} \int_{\mathfrak{X}}\left[p_{k, n}(x, y)-p_{\frac{k}{n}}(x, y)\right] \Psi_{A\left(\frac{k}{n}\right)^{\delta}}(x, y) \mu_{n}(d y)\right|, \\
\Delta_{n}^{2}(x, A, s, t)=\mid \frac{1}{n} \sum_{k=1}^{n n(t-s)[-1} \int_{\mathfrak{X}} p_{\frac{k}{n}}(x, y) \Psi_{A\left(\frac{k}{n}\right)^{\delta}}(x, y) \mu_{n}(d y) \\
\quad-\int_{0}^{t-s} \int_{\mathfrak{X}} p_{r}(x, y) \Psi_{A r^{\delta}}(x, y) \mu_{n}(d y) d r \mid, \\
\Delta_{n}^{3}(x, A, s, t)=\left|\int_{0}^{t-s} \int_{\mathfrak{X}} p_{r}(x, y) \Psi_{A r^{\delta}}(x, y)\left[\mu_{n}(d y)-\mu(d y)\right] d r\right| .
\end{gathered}
$$

Put

$$
\Delta_{n}^{i}(A)=\sup _{x \in \mathfrak{X}, s \leq t \leq T} \Delta_{n}^{i}(x, A, s, t), \quad i=1,2,3 .
$$


Since $\Psi_{r}(x, y) \in[0,1]$ and $\left\{\Psi_{r}(x, y) \neq 0\right\} \subset\{y \in B(x, 2 r)\}$, we have

$$
\begin{aligned}
\Delta_{n}^{1}(A) & \leq \frac{1}{n} \sum_{k=1}^{] n T[-1} \alpha_{k}\left(\frac{n}{k}\right)^{\gamma} \mu_{n}\left(B\left(x, 2 A\left(\frac{k}{n}\right)^{\delta}\right)\right) \\
& \leq C_{\theta}(2 A)^{\theta} \cdot \frac{1}{n} \sum_{k=1}^{] n T[-1} \alpha_{k}\left(\frac{k}{n}\right)^{\delta \theta-\gamma} \rightarrow 0, \quad n \rightarrow+\infty,
\end{aligned}
$$

by the Toeplitz theorem.

The function

$$
(r, x, y) \mapsto p_{r}(x, y) \Psi_{r}(x, y)
$$

is uniformly continuous on $\left[r_{0},+\infty\right) \times \mathfrak{X}^{2}$ if $r_{0}>0$. Thus an estimate, similar to (4.7), implies the uniform convergence (with respect to $x \in \mathfrak{X}$ and $(s, t) \in \mathbb{T}$ ) to zero of the sequence

$$
\left|\frac{1}{n} \sum_{k=\left[r_{0} n\right]+1}^{] n(t-s)[-1} \int_{\mathfrak{X}} p_{\frac{k}{n}}(x, y) \Psi_{A\left(\frac{k}{n}\right)^{\delta}}(x, y) \mu_{n}(d y)-\int_{r_{0}}^{t-s} \int_{\mathfrak{X}} p_{r}(x, y) \Psi_{A r^{\delta}}(x, y) \mu_{n}(d y) d r\right|
$$

(note that $\sup _{n} \mu_{n}(\mathfrak{X})<+\infty$, since the measures $\mu_{n}$ weakly converge to $\mu$ ). The same reasoning leads to the bound

$$
\begin{aligned}
\limsup _{n \rightarrow+\infty} \Delta_{n}^{2}(A) \leq & \limsup _{n \rightarrow+\infty}\left[\frac{1}{n} \sum_{k=1}^{\left[r_{0} n\right]} C_{\gamma}\left(\frac{n}{k}\right)^{\gamma} C_{\theta}\left(2 A\left(\frac{k}{n}\right)^{\delta}\right)^{\theta}\right] \\
& +\limsup _{n \rightarrow+\infty}\left[\int_{0}^{r_{0}} C_{\gamma} r^{-\gamma} C_{\theta}\left(2 A r^{\delta}\right)^{\theta} d r\right] \\
= & B_{7}(A)\left(r_{0}\right)^{\delta \theta-\gamma+1}
\end{aligned}
$$

for $A>c_{\theta}$ and arbitrary $r_{0}>0$ (the precise value of the constant $B_{7}(A)$ does not matter). This implies the convergence

$$
\Delta_{n}^{2}(A) \rightarrow 0, \quad n \rightarrow+\infty .
$$

Finally, the weak convergence of $\mu_{n}$ to $\mu$ and the first part of condition (44) imply that

$$
I_{n}(A, t) \equiv \sup _{x \in \mathfrak{X}}\left|\int_{\mathfrak{X}} p_{t}(x, y) \Psi_{A r^{\delta}}(x, y)\left[\mu_{n}(d y)-\mu(d y)\right]\right| \rightarrow 0, \quad n \rightarrow+\infty,
$$

for all $t$. Since $I_{n}(A, t) \leq C_{\gamma} t^{-\gamma} \cdot C_{\theta}\left(2 A t^{\delta}\right)^{\theta}$, the Lebesgue dominated convergence theorem yields

$$
\Delta_{n}^{3}(A) \rightarrow 0, \quad n \rightarrow+\infty .
$$

Now we obtain from bounds (4.5)-(4.9) that

$$
\limsup _{n \rightarrow+\infty} \sup _{x \in \mathfrak{X}, s \leq t \leq 1}\left|f_{n}^{s, t}(x)-f^{s, t}(x)\right| \leq\left(B_{5}(T)+B_{6}(T)\right) A^{-\beta / 2}, \quad A>\max \left(c_{\theta}, A_{2}\right) .
$$

Letting $A \rightarrow+\infty$ we get relation (4.1), and this completes the proof of Theorem 1

\section{EXAMPLE}

Assume that $\xi_{n}, n \geq 1$, is a sequence of independent identically distributed random variables with density such that the normalized sums

$$
S_{n} \stackrel{\text { def }}{=} n^{-1 / \alpha} \sum_{k=1}^{n} \xi_{k}
$$


weakly converge to a stable random variable with the characteristic function

$$
\varphi_{0}(\lambda)=\exp \left(i a \lambda-b|\lambda|^{\alpha}(1+i \beta \omega(\lambda, \alpha))\right)
$$

where $0<\alpha \leq 1,-1 \leq \beta \leq 1, a, b \in \mathbb{R}$, and

$$
\omega(\lambda, \alpha)= \begin{cases}\frac{2}{\pi} \operatorname{sgn} \lambda \ln |\lambda|, & \alpha=1 \\ \frac{2}{\pi} \operatorname{sgn} \lambda \tan \frac{\pi \alpha}{2}, & \alpha \neq 1\end{cases}
$$

We construct a sequence of processes $X_{n}(t)$ as follows. First we put

$$
X_{n}(k / n)=\frac{1}{n^{1 / \alpha}} \sum_{i=1}^{k} \xi_{i}, \quad k=0, \ldots, n,
$$

for $t \in[0,1]$. Then we use the linear interpolation to determine $X_{n}(t)$ for other $t \in[0,1]$. In Proposition 2 below we assume that the distribution of $\xi_{1}$ has bounded density on $\mathbb{R}$. Then Gnedenko's theorem ([7, Theorem 4.3.1]) implies that the densities of the random variables $S_{n}$ converge uniformly to the density of a stable distribution with index $\alpha$. Condition (1) of Theorem 1 is proved for this case in the paper [1, where the approximation in the Markov sense is applied to random polygonal lines for random variables belonging to the domain of attraction of a stable law.

Denote by $K$ the Cantor set (that is, the set of points in the interval $(0,1)$ whose ternary numeral does not contain digit 1 ). Put

$$
K_{\varepsilon} \stackrel{\text { def }}{=}\{x \in \mathbb{R}|\exists y \in K:| x-y \mid \leq \varepsilon\} .
$$

Let $\lambda_{1}(\cdot)$ be Lebesgue measure in the line, and let $h(n) \stackrel{\text { def }}{=}\left[\log _{3} \frac{n}{2}\right]$. Consider the functionals $\phi_{n}^{s, t}$ of the form (2.1), where the function $g_{n}$ is such that $n g_{n}(x)=a(n) \chi_{\left\{K_{r_{n}}\right\}}(x)$; that is, consider the functionals

$$
\phi_{n}^{s, t}(Y) \stackrel{\text { def }}{=} \sum_{k: s \leq k / n<t} a(n) \chi_{\left\{K_{r_{n}}\right\}}\left(Y\left(\frac{k}{n}\right)\right)
$$

where $r_{n} \stackrel{\text { def }}{=} n^{-1}$ and

$$
a(n) \stackrel{\text { def }}{=} \frac{1}{n}\left(\lambda_{1}\left\{K_{r_{n}}\right\}\right)^{-1}=\frac{1}{n}\left(2^{h(n)}\left(\frac{1}{3^{h(n)}}+\frac{2}{n}\right)\right)^{-1} .
$$

The meaning of these functionals for a fixed $n$ is that they represent, up to a constant factor, the fraction of time spent by the random walk in the $r_{n}$-neighborhood of the Cantor set.

In the following assertion we prove that the random polygonal lines corresponding to functionals (5.2) converge to some $W$-functional of the limit process.

Proposition 2. Assume that independent identically distributed random variables $\xi_{n}$ have a bounded density and, for some $\alpha \in(1-\ln 2 / \ln 3,1]$, the normalized sums

$$
S_{n} \stackrel{\text { def }}{=} n^{-1 / \alpha} \sum_{k=1}^{n} \xi_{k}
$$

weakly converge to a stable random variable whose characteristic function is given by equality (5.1). Then the random polygonal lines $\psi_{n}$ constructed from the functionals (5.2) converge in distribution in the space $C\left(\mathbb{T}, \mathbb{R}^{+}\right)$, that is,

$$
\psi_{n}\left(X_{n}\right) \Rightarrow \phi(X)
$$

where $\phi(X)$ is a $W$-functional of the stable process $X$. It is natural to view the functional $\phi(X)$ as the local time of the process $X$ in the Cantor set $K$. 
We use the main result of this paper, Theorem 1, to prove Proposition 2, We already mentioned that condition (1) of Theorem 1 holds. The relation

$$
p_{t}(x)=t^{1 / \alpha} p_{1}\left(x t^{1 / \alpha}\right)
$$

(called the automodel property of a stable process) implies condition (4) of Theorem 1 for $\gamma=\alpha^{-1}$, since the density $p_{1}(\cdot)$ is bounded. The Gnedenko theorem and the second assumption of Proposition 2 imply condition (5), while condition (3) follows from the following estimate:

$$
\frac{1}{n}\left(2^{h(n)}\left(\frac{1}{3^{h(n)}}+\frac{2}{n}\right)\right)^{-1} \leq 2^{-h(n)}\left(\frac{n}{n / 2}+2\right)^{-1} \leq \frac{1}{4} \cdot 2^{-h(n)} \rightarrow 0, \quad n \rightarrow \infty .
$$

Now we check condition (6). Recall that the measures mentioned in Theorem 1 are such that in the case under consideration we have

$$
\mu_{n}(D)=a(n) \lambda_{1}\left\{K_{r_{n}} \cap D\right\} \quad \text { for all } D \in \mathcal{B}(\mathbb{R}),
$$

where $\lambda_{1}$ is Lebesgue measure on the line. Let $R$ be a number such that

$$
\frac{1}{3^{h(n)}}+\frac{1}{n}<R<1
$$

and let $H(R) \stackrel{\text { def }}{=}-\left[\log _{3} R\right]$. Then

$$
\frac{1}{3^{H(R)+1}}<R \leq \frac{1}{3^{H(R)}} .
$$

Thus

$$
\begin{aligned}
\mu_{n}((0, R)) & =n \cdot a(n) \lambda_{1}\left\{K_{r_{n}} \cap(0, R)\right\} \leq n \cdot a(n) \lambda_{1}\left\{K_{r_{n}} \cap\left(0,3^{-H(R)}\right)\right\} \\
& \leq n \cdot a(n) 2^{h(n)-H(R)} \cdot \frac{\lambda_{1}\left\{K_{r_{n}}\right\}}{2^{h(n)}}=2^{-H(R)}=R^{-(\ln 2 / \ln R) H(R)} \\
& \leq R^{-(\ln 2 / \ln R)(-\ln R / \ln 3+1)} \leq R^{\ln 2 / \ln 3} .
\end{aligned}
$$

If $R<1$ and $0<R<3^{-h(n)}+n^{-1}$, then

$$
\mu_{n}((0, R))=R n \cdot a(n) \leq R \leq R^{\ln 2 / \ln 3} .
$$

Moreover one can choose $\theta=\ln 2 / \ln 3$.

In a similar way we prove the weak convergence of $\mu_{n}$ to the measure $\mu$ constructed from the Cantor function, which is known to be a distribution function (see [6, Chapter 5 , $\S 8])$. Let

$$
\mu_{n}(x) \stackrel{\text { def }}{=} \mu_{n}([0, x]), \quad \mu(x) \stackrel{\text { def }}{=} \mu([0, x]), \quad x \in[0,1] .
$$

Since the distribution function $\mu(\cdot)$ is continuous, we need to check the pointwise convergence of $\mu_{n}(\cdot)$. We prove that for all $x \in\left(\frac{1}{3}, \frac{2}{3}\right)$,

$$
\mu_{n}(x) \rightarrow \mu(x) .
$$

If $n_{0}$ is chosen so that $x \notin K_{r_{n}}$ for $n \geq n_{0}$, then

$$
\mu_{n}(x)=n a(n) 2^{h(n)-1} \frac{\lambda_{1}\left\{K_{r_{n}}\right\}}{2^{h(n)}}=\frac{1}{2}=\mu(x)
$$

for $n \geq n_{0}$. Similarly we prove that

$$
\mu_{n}(x) \rightarrow \mu(x), \quad n \rightarrow \infty,
$$

for all $x \in[0,1] \backslash K$.

Since the functions $\mu_{n}(\cdot)$ are monotone, $\mu(\cdot)$ also is monotone and continuous. The set $[0,1] \backslash K$ is everywhere dense in $[0,1]$, whence the pointwise convergence follows for all points of the interval $[0,1]$. Thus condition (6) holds. 
If $\xi$ is an arbitrary random variable and $C>0$ is an arbitrary constant, then

$$
\mathrm{P}[|\xi| \geq C] \leq 7 C \int_{0}^{1 / C}|1-\varphi(t)| d t
$$

where $\varphi: t \rightarrow \mathrm{E}\left[e^{i t \xi}\right]$ is the characteristic function of the random variable $\xi$ (see $[9$, Lemma $3, \S 3$, Chapter 3]). Denote by $\varphi_{1}(\cdot)$ the characteristic function of the random variable $\xi_{1}$. According to Theorem 1.4 in [4,

$$
\begin{aligned}
\exists \varepsilon \in(0,1), a, b>0: \quad \forall \lambda,|\lambda|<\varepsilon \\
\ln \varphi_{1}(\lambda)=a i \lambda-b|\lambda|^{\alpha}(1+i \beta \omega(\lambda, \alpha))(1+r(\lambda)), \quad r(\lambda) \rightarrow 0, \lambda \rightarrow 0 .
\end{aligned}
$$

Thus we may assume that

$$
|b| \cdot|1+i \beta \omega(\lambda, \alpha)| \cdot|\lambda|^{\alpha}|1+r(\lambda)|<\frac{1}{2} \quad \text { and } \quad|a \lambda|<\frac{1}{2}
$$

for $\lambda$ such that $|\lambda|<\varepsilon$.

Put

$$
r_{1}(\cdot) \stackrel{\text { def }}{=} \operatorname{Re}(r(\cdot)), \quad r_{2}(\cdot) \stackrel{\text { def }}{=} \operatorname{Im}(r(\cdot)) .
$$

If $c_{0}>1 / \varepsilon$ is fixed, then

$$
\begin{aligned}
\mathrm{P}\left\{\frac{S_{n}}{n^{1 / \alpha}}>c\right\} & \leq 7 c \int_{0}^{1 / c}\left|1-\varphi_{1}^{n}\left(\lambda n^{-1 / \alpha}\right)\right| d \lambda \\
& \leq 7 c \int_{0}^{1 / c}\left|1-e^{\left(a \cdot i \lambda \cdot n^{1-1 / \alpha}-b(1+i \beta \omega(\lambda, \alpha))|\lambda|^{\alpha}\left(1+r\left(\lambda \cdot n^{-1 / \alpha}\right)\right)\right)}\right| d \lambda
\end{aligned}
$$

for all $c>c_{0}$.

Let

$$
h \stackrel{\text { def }}{=} \max \left(\tan \pi \frac{\alpha}{2}, \frac{2}{\pi}\right) .
$$

If $x, y \in \mathbb{R}$ are two arbitrary real numbers such that $|x|+|y|<1$, then

$$
\left|e^{x+i y}-1\right| \leq\left|e^{x+i y}-e^{x}\right|+\left|1-e^{x}\right| \leq e\left|e^{i y}-1\right|+e|x| \leq 2 e(|x|+|y|) .
$$

This inequality allows us to estimate the last term in (5.4). First we consider the case of $\alpha<1$ :

$$
\begin{aligned}
14 e c & \left.\int_{0}^{c^{-1}}\left|a \cdot i \lambda \cdot n^{1-1 / \alpha}-b(1+i \beta \omega(\lambda, \alpha))\right| \lambda\right|^{\alpha}\left(1+r\left(\lambda \cdot n^{-1 / \alpha}\right)\right) \mid d \lambda \\
& \leq 14 e c \int_{0}^{c^{-1}}\left(|a| \cdot|\lambda|+2|b| h|\lambda|^{\alpha}\right) d \lambda \\
& \leq 28 e c \int_{0}^{c^{-1}}(|a|+|b h|) \lambda^{\alpha} d \lambda \\
& \leq 28 e c^{-\alpha}(|a|+h|b|) .
\end{aligned}
$$


Now let $\alpha=1$ and $c>e$. Then

$$
\begin{aligned}
14 e c & \int_{0}^{c^{-1}}|a \cdot i \lambda-b(1+i \beta \omega(\lambda, 1))| \lambda\left|\left(1+r\left(\lambda \cdot n^{-1}\right)\right)\right| d \lambda \\
& \leq 14 e c \int_{0}^{c^{-1}}(|a| \cdot|\lambda|+2|b| h(1+|\ln \lambda|)|\lambda|) d \lambda \\
& \leq 28 e c \int_{0}^{c^{-1}}(|a|+|b h|(1+|\ln \lambda|)) \lambda d \lambda \\
& \leq 28 e c^{-1}(|a|+h|b|)+28 e|b| h c \int_{0}^{c^{-1}}(-\lambda \ln \lambda) d \lambda \\
& \leq 28 e c^{-1}(|a|+h|b|)+28 e|b| h c\left(\frac{1}{2} c^{-2} \ln c+\frac{1}{4} c^{-2}\right) \\
& \leq 56 e c^{-1 / 2}(|a|+h|b|) .
\end{aligned}
$$

By assumption, $\alpha \in(1-\ln 2 / \ln 3,1]$. Thus there exists $\delta$ such that

$$
(1 / \alpha-1) \frac{\ln 3}{\ln 2}<\delta<1 / \alpha .
$$

If the parameters $\gamma, \delta$, and $\theta$ are chosen as indicated above, then

$$
\delta \theta+1-\gamma=\delta \frac{\ln 2}{\ln 3}+1-\frac{1}{\alpha}>0 ;
$$

that is, condition (7) of Theorem 1 holds. Condition (2) holds if

$$
\mathrm{P}\left(\frac{S_{k}}{n^{1 / \alpha}}>A\left(\frac{k}{n}\right)^{\delta}\right) \leq C A^{-\beta}\left(\frac{k}{n}\right)^{\varepsilon}
$$

for some $\beta>0$ and $\varepsilon \in(0,1)$. The last condition holds, indeed, since, as we have proved above,

$$
\mathrm{P}\left(\frac{S_{k}}{n^{1 / \alpha}}>A\left(\frac{k}{n}\right)^{\delta}\right)=\mathrm{P}\left(\frac{S_{k}}{k^{1 / \alpha}}>A\left(\frac{k}{n}\right)^{\delta-1 / \alpha}\right) \leq[28 e(|a|+h|b|)] A^{-\alpha}\left(\frac{k}{n}\right)^{(1-\alpha \delta)}
$$

for sufficiently large $A$ and $\alpha \in(1-\ln 2 / \ln 3,1)$, and

$$
\mathrm{P}\left(\frac{S_{k}}{n}>A\left(\frac{k}{n}\right)^{\delta}\right) \leq[56 e(|a|+h|b|)] A^{-1 / 2}\left(\frac{k}{n}\right)^{(1-\delta)}
$$

for $\alpha=1$.

We have checked all the assumptions of Theorem 1, and this proves Proposition 2.

Remark 1. The paper 2] contains two results on the sufficient conditions for the convergence of characteristics. The feature of the example considered in Section 5 is that neither of the results of [2] can be applied. The crucial assumption of Theorem 2 in 22 is that the trajectories are Hölderian. This assumption does not hold for the above-mentioned example, since the trajectories of the process $X$ are discontinuous almost surely. Theorem 3 of [2] also cannot be applied, since $\gamma=\alpha^{-1} \geq 1$. Therefore Proposition 2 shows that Theorem 1 of this paper is a nontrivial extension of the preceding results of [2]. 


\section{BIBLIOGRAPHY}

1. A. M. Kulik, Markov approximation of stable processes by random walks, Theory Stoch. Proccess. 12(28) (2006), no. 1-2, 87-93. MR2316289 (2008j:60082)

2. A. M. Kulik and Yu. N. Kartashov, Invariance principle for additive functionals of Markov chains, arXiv:0704.0508v1.

3. E. B. Dynkin, Markov Processes, Fizmatgiz, Moscow, 1963; English transl., vols. I and II, Academic Press and Springer-Verlag, New York and Berlin-Göttingen-Heidelberg, 1965. MR.0193670(33:1886)

4. A. N. Borodin and I. A. Ibragimov, Limit theorems for functionals of random walks, Trudy Mat. Inst. Steklov., vol. 195, 1994; English transl. in Proc. Steklov Inst. Math. 195 (1995), no. 2. MR $1368394(97 \mathrm{j}: 60140)$

5. W. Feller, An Introduction to Probability Theory and its Applications, 2nd ed., vol. 2, John Wiley \& Sons, New York-London-Sydney, 1971. MR0270403 (42:5292)

6. A. N. Kolmogorov and S. V. Fomin, Elements of the Theory of Functions and Functional Analysis, 6th ed., "Nauka", Moscow, 1989; English transl. of 1st ed., Graylock, Rochester, NY, 1957. MR1025126 (90k:46001) MR0085462 (19:44d)

7. I. A. Ibragimov and Yu. V. Linnik, Independent and Stationary Sequences of Random Variables, "Nauka", Moscow, 1965; English transl., Wolters-Noordhoff, Groningen, 1971. MR0322926 $(48: 1287)$

8. A. V. Skorokhod, Lectures on the Theory of Stochastic Processes, "Lybid", Kyiv, 1990; English transl., VSP and TViMS Scientific Publishers, Utrecht and Kiev, 1996. MR 1452108 (99d:60001)

9. A. N. Shiryaev, Probability, "Nauka", Moscow, 1980; English transl., Springer-Verlag, New York, 1996. MR1368405 (97c:60003)

Department of Probability Theory and Mathematical Statistics, Faculty for Mechanics and Mathematics, National Taras Shevchenko University, Academician Glushkov Avenue 6 , KYIV 03127, UKRAINE

E-mail address: kartashov-y@yandex.ru

Received 17/MAY/2007

Translated by S. KVASKO 